\title{
HISTORIA CLÍNICA INFORMATIZADA DE ENFERMERÍA EN EL DEPARTAMENTO DE TORREVIEJA SALUD COMO INSTRUMENTO DE GESTIÓN DE CALIDAD
}

\section{ELECTRONIC NURSING CLINICAL HISTORY IN THE DEPARTMENT OF TORREVIEJA SALUD HOSPITAL, SPAIN, AS AN INSTRUMENT OF QUALITY MANAGEMENTI}

*Cerezo Sanmartín M, "Gutiérrez Aranda, JM. "**Perán Artero, S. **Gómez Gonzálvez, G., "Carrasco Gutiérrez, P.

*Enfermera. Supervisora Formación y Calidad. **Enfermera. Dirección Operaciones de Enfermería. ***Enfermera Responsable Formación HCE. ****Enfermero. Supervisor Especialidades Médicas. Departamento de Torrevieja. Hospital de Torrevieja. Alicante.

Palabras clave: Historia clínica informatizada, sistemas de información, enfermería, trabajo en equipo .

Keywords: Electronic Clinical History, Teamwork, and Nursing

\section{RESUMEN}

En la sociedad de la información del siglo XXI, la historia clínica electrónica (HCE) es un hecho que no está por igual desarrollado en todos los países. En el Departamento de Salud de Torrevieja, Alicante, se trabaja con un modelo de HCE único que engloba a todas las áreas incluida enfermería y en un nivel avanzado de operabilidad en cuanto a manejo de datos.

Objetivos:

1. - Desarrollo de los sistemas de información con el fin de facilitar la comunicación e intercambio de datos entre los distintos profesionales de atención especializada y primaria que componen el Departamento de Salud.

2. - Aunar y optimizar los conocimientos propios de los profesionales enfermeros e informáticos, a través del trabajo en conjunto.

Metodología: Para llevar a cabo esta mejora en los registros de enfermería se ha utilizado la aplicación informática, "Florence", modelo de Historia Clinica Electrónica única y global para el Departamento de Salud de Torrevieja que consta de dos entornos: Florence Gestión (área financiera- contable y área de recursos humanos) y Florence clínico (área clínico-asistencial). 
A través del trabajo en equipo de Informáticos y profesionales de enfermería que conocen el sistema e inquietudes de los profesionales se obtiene un producto doblemente valorado en cuanto a metodología enfermera y suma de los últimos conocimientos tecnológicos.

Conclusiones: Los registros de enfermería se consideran un instrumento efectivo para reflejar toda la información necesaria para poder coordinar y proporcionar unos cuidados de enfermería de máxima calidad al estar almacenados de forma integral. Por otro lado se asegura la disponibilidad de toda la información de los procesos salud-enfermedad de un individuo, asegurando la confidencialidad.

\section{ABSTRACT}

In the information society of the $21^{\text {st }}$ century, it is a fact that the Electronic Clinical History (ECH) is not developed in every country. In Torrevieja Salud Health Department, Alicante (Spain), they work with a unique $\mathrm{ECH}$ that includes all divisions, including the nursing ones and an advanced operability level in regards to data management.

\section{Objectives:}

1.- To develop all the information sources to facilitate the communication at all levels of care, so any professional in the community or at the hospital can retrieve information about the patient.

2. - To gather the nursing staff and computer technicians knowledge by working together.

Methodology: In order to improve the nursing records, they have used a computer program named "FLORENCE", which is a model of a unique clinical history for the Health Department of Torrevieja. Florence is divided in two environments: Florence Gestión (accountant-financial, and HH RR scopes) and FLORENCE (clinical assitance scope).

This program comes as the result of the teamwork from IT and nursing staff who are knowledgeable of the concerns of the professionals. A product with double value because it combines the nursing methodology and state-of-art technological advances.

Conclusions: Nursing records are valued as an effective instrument to empower the continuation of nursing care and coordinate that care at the best quality, as they are registered in a unique and single program. On the other hand, it ensures constant availability of all the information of the health-disease episodes of a patient, and most importantly, the information is kept confidential.

\section{INTRODUCCION}

En la sociedad de la información del siglo $X X I$, los modelos e instauración de Historia Clínica Electrónica (HCE) completa son desiguales, de hecho en el país de la informática por antonomasia, USA, la Sociedad de Información Sanitaria y Sistemas de Gestión: Himss (1) por sus siglas en inglés, publicaba un informe en diciembre 2008 argumentando que tan sólo un $6 \%$ de los centros sanitarios reportaban una adopción completa de las aplicaciones informáticas, mientras que un $67 \%$ necesitaban añadir una o dos aplicaciones para alcanzar el umbral adecuado en cuanto a funcionalidad e interoperabilidad de la HCE.

En España nos encontramos en las puertas de esos niveles, tanto que en el Departamento de Torrevieja, que proporciona una asistencia integral a sus usuarios a través de sus 10 centros de salud y del propio Hospital de Torrevieja, hospital comarcal con 269 habitaciones individuales y amplia cartera de servicios, la idea inicial de "un hospital sin papeles"es una realidad consolidada.

En el bloque de la HCE referente a Enfermería, hemos ido desarrollando una serie de registros donde los profesionales dejen constancia de todas las actividades que llevan a cabo en el proceso de cuidar teniendo a su vez un acceso inmediato a toda la información de un paciente, sin que importe donde se encuentre. 


\section{OBJETIVOS}

1. - Desarrollo de los sistemas de información con el fin de facilitar la comunicación e intercambio de datos entre los distintos profesionales de atención especializada y primaria que componen el Departamento de Salud.

2. - Aunar y optimizar los conocimientos propios enfermeros e informáticos, a través del trabajo en equipo de estos profesionales.

\section{METODOLOGÍA}

Según el estándar industrial Emram (2), que se desarrolló en 2005 para medir el progreso en la implantación de la HCE por las organizaciones sanitarias, éste contempla ocho niveles, desde el cero, en el que los sistemas departamentales complementarios de radiología, laboratorio y farmacia no están implantados, hasta el siete, donde papel e historia clínica son irreconciliables, y se cuenta con capacidad de almacenamiento y minería de datos como para analizar y mejorar protocolos y enviar información resumida.

La HCE del Departamento de Torrevieja, "Florence", se considera una historia clínica global que integra toda la actividad asistencial e incluye en ella todas las áreas del hospital: Bloque quirúrgico, Hospitalización, Urgencias, Consultas Externas, Atención Primaria, Gestión de pacientes, Medios de Diagnóstico, Facturación, Costes....

Esta aplicación se ha desarrollado con las últimas tecnologías, incorporando las herramientas más novedosas que proporciona la industria. Desde el punto de vista funcional, la aplicación se ha proyectado según las necesidades que han ido surgiendo desde la apertura del hospital contando con profesionales de todas las áreas que han participado en su crecimiento como historia clínica única en el Departamento.

\section{FLORENCE se caracteriza por:}

- Calidad de registro

- Facilidad de acceso a la información, siendo ésta, ordenada, limpia y legible

- Rapidez en su uso.

-Fomentar el trabajo en equipo, al permitir el acceso compartido a exploraciones y tratamientos.

- Seguridad y protección de la información

- Permitir la medición de indicadores relativos a la calidad asistencial, con fines investigadores

- Impulsar LA ATENCIÓN INTEGRAL entre Atención primaria y especializada.

La historia se encuentra ordenada cronológicamente por episodios asistenciales, y compuesta por una serie de documentos que se dividen en bloques.( Fig. 1).

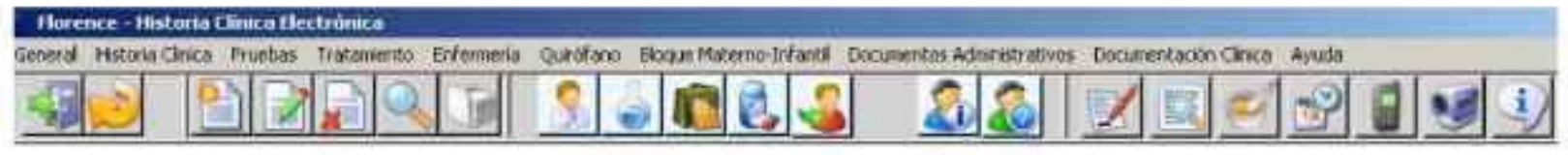

Fig.1 
Dentro del Bloque de enfermería cabe destacar la implantación en todo el hospital de un modelo de VALORACION INICIAL (Fig.2) basado en los patrones funcionales de salud de Marjory Gordon (3) y la utilización de un lenguaje común NANDA, NIC y $\operatorname{NOC}(4,5,6)$ para la elaboración de un plan de cuidados personalizado para cada paciente con el fin de facilitar la gestión de los cuidados y establecer registros que nos facilitarán mayor información sobre el paciente.

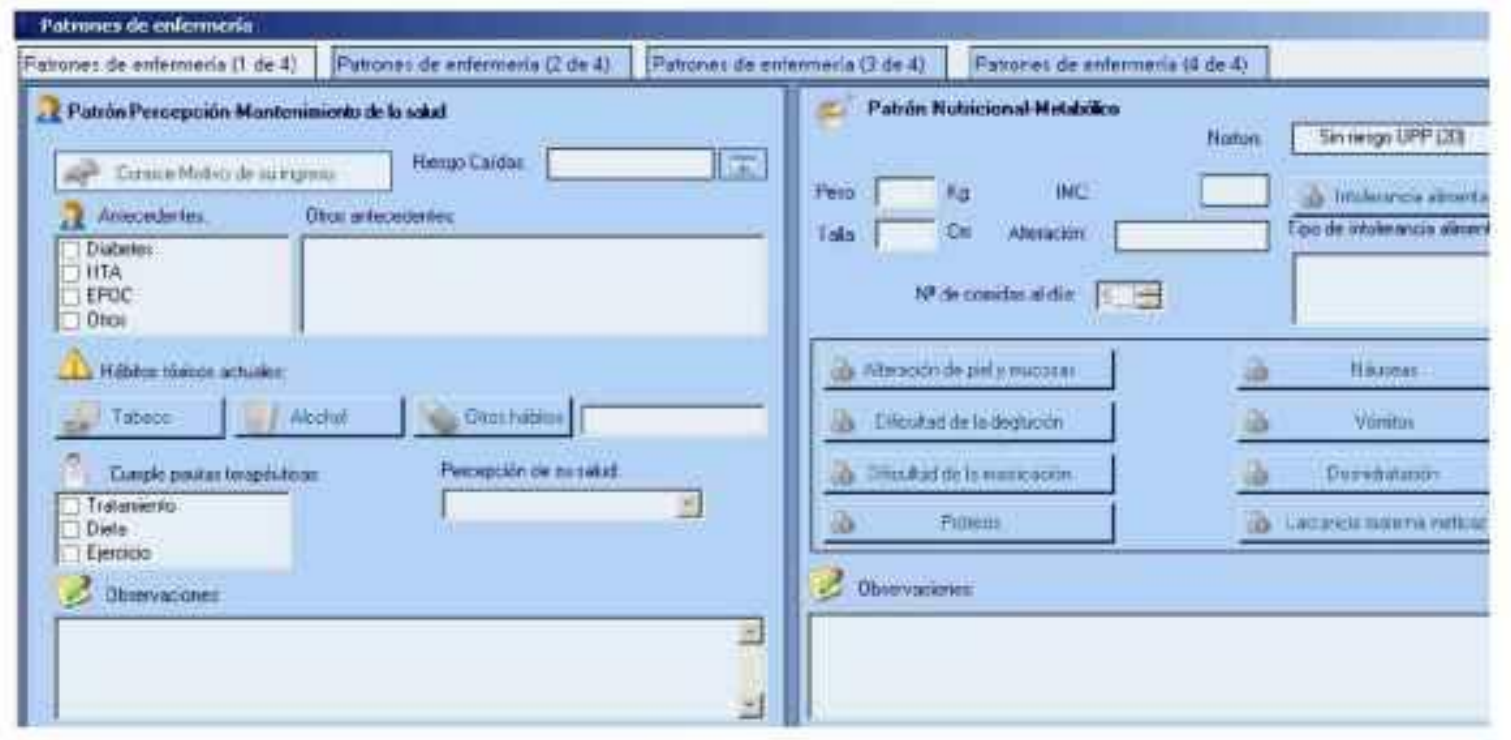

Fig. 2: Ejemplo de una de las pantallas de la valoración inicial

La valoración inicial. (Fig. 3) está estructurada de tal manera que tras el registro de todos los patrones la aplicación detecta los posibles problemas de salud del paciente y le sugiere al usuario una serie de diagnósticos y actividades posibles, de los cuales la enfermera escogerá los que considere oportunos.

La valoración se debe de realizar en las primeras 24 horas del ingreso del paciente 
LACTANCIAMRTERNA EFICA?

MANEJO INEFECTIVO DEL REGIMEN TERAPEUTICO

TRASTORINO DE LA PERCEFCION SEIASORIOL (VSUAL AUDITNA, OUSTATMA, TACTIC)

Actividades

Apous en la tamat de decitiones

Educacien Baritaria

Enteĥanza

Fomento de habitos raludables

Identricicoítn de riesgo:

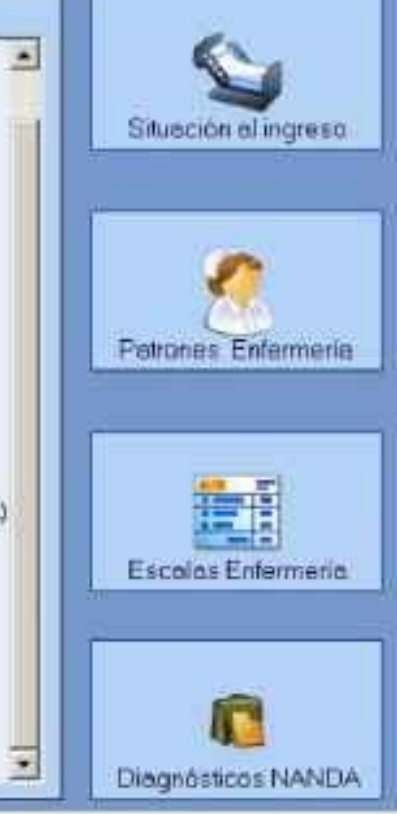

Fig. 3

Se incorporan en la valoración escalas de salud (Fig. 4) con resultados e interpretaciones, por ejemplo la escala Downtown (7) que en función de su valor, activa las diferentes medias de prevención y cuidados para evitar caídas hospitalarias, indicador de calidad del servicio de enfermería y seguridad del paciente.

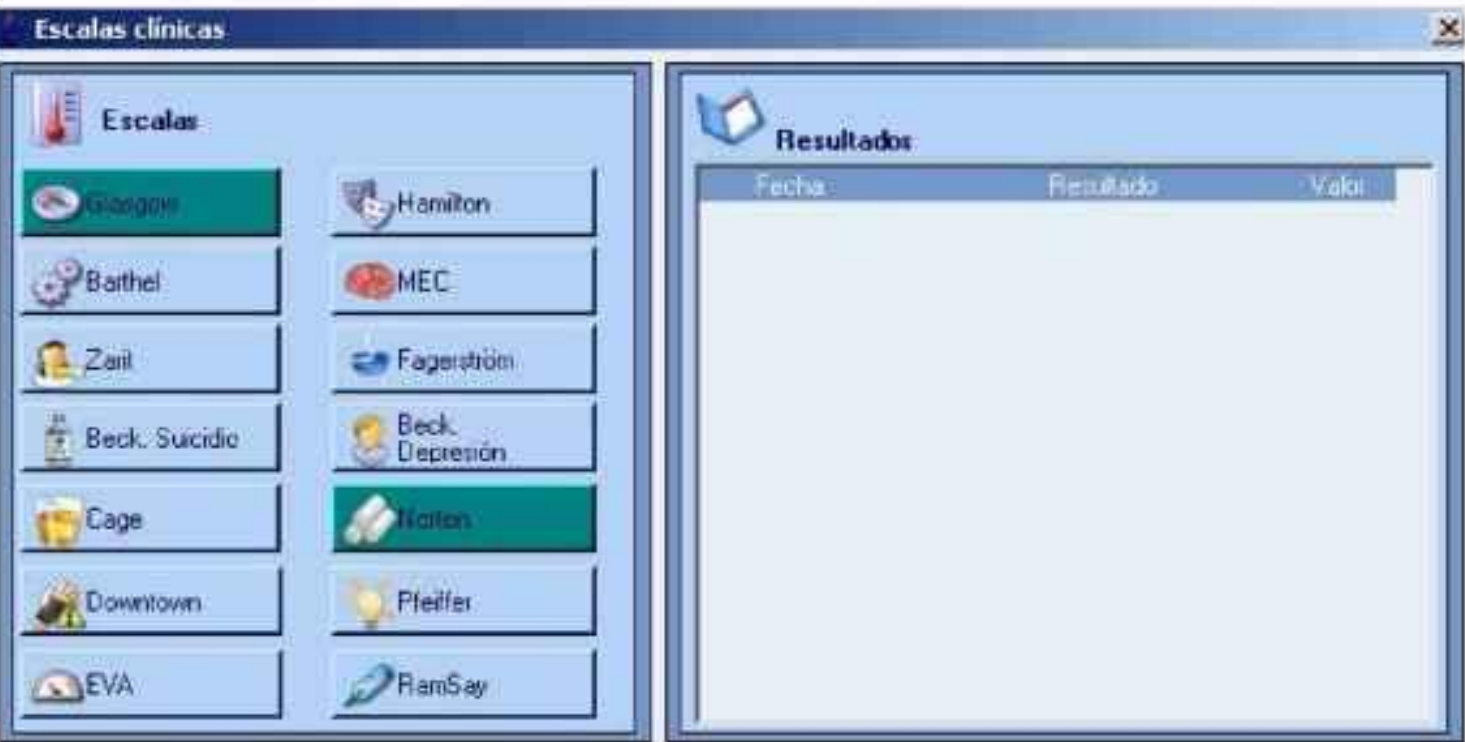

Fig.4

La evolución del paciente (fig.5) es reflejada diariamente en una pantalla con acceso a diferentes formularios donde se recogen todos los cuidados (8) de enfermería llevados a cabo en el paciente, así como la medicación administrada, seguimiento de heridas, gráfica de constantes, Balances hídricos, control de pruebas, control de dietas, etc 

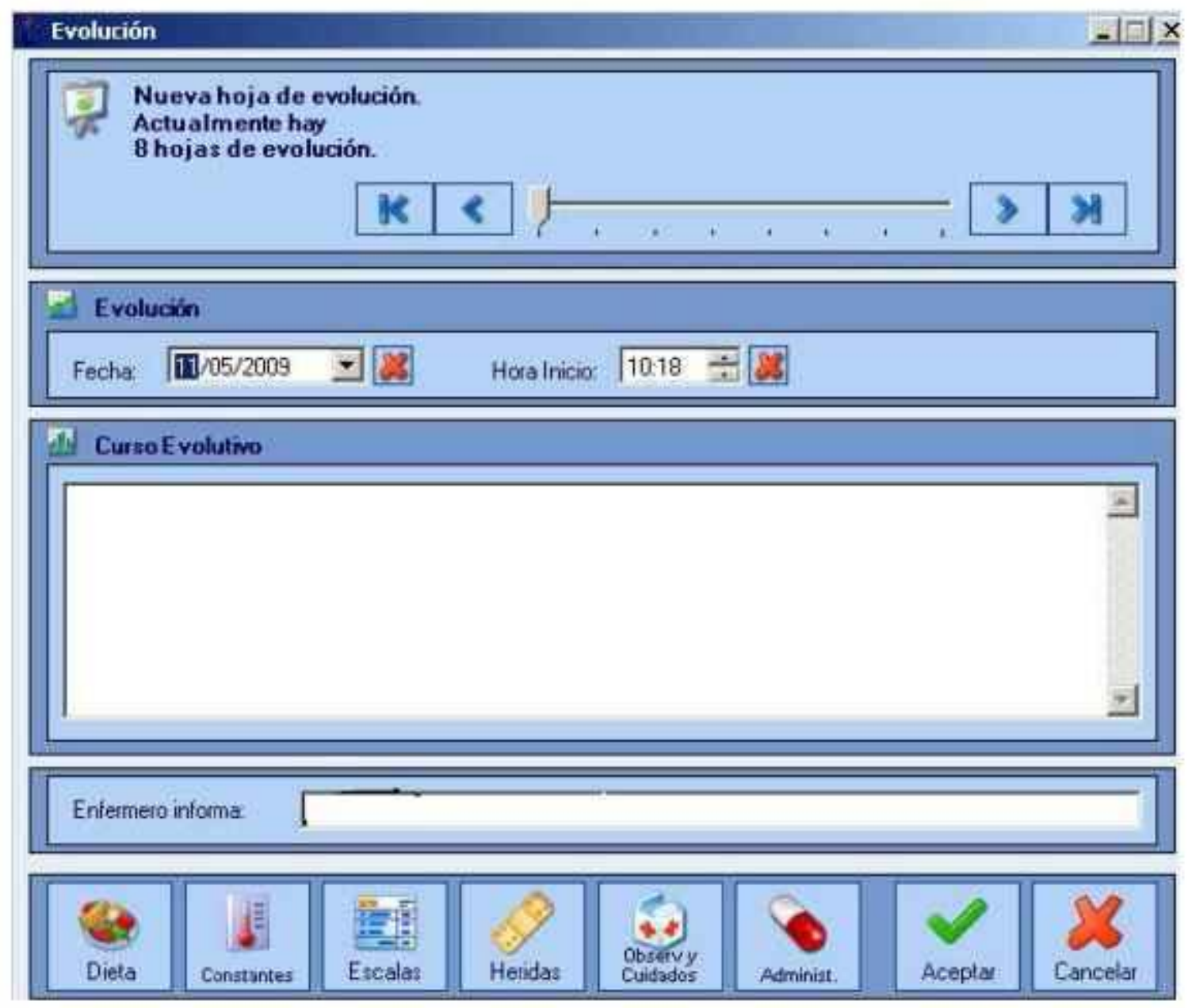

Fig.5

Para garantizar la continuidad de los cuidados del paciente tras el alta, se realiza en Florence un INFORME DE ALTA DE ENFERMERÍA en el que se refleja un resumen de la situación del paciente, plan de cuidados (9), recomendaciones y los problemas de salud que deben solucionarse tanto en el domicilio como atención primaria, Hospital a Domicilio $\mathrm{u}$ otras unidades o centros sanitarios a las que sea trasladado el paciente.

\section{RESULTADOS}

Tras dos meses de instauración de la nueva metodología de trabajo, se ha conseguido el $100 \%$ de cumplimentación de la valoración inicial, los profesionales indican que en quince minutos tienen realizada la valoración de Enfermería. La pantalla de evolución ha supuesto una mejora con respecto a la anterior de 2006, puesto que ahora tienen un campo de texto más extenso.

El alta de Enfermería se realiza a los pacientes que son más proclives de cuidados de enfermería y seguimiento fuera del área hospitalaria convencional. Se suele dar una copia en formato papel para el paciente, mientras que el registro original queda en la HCE, para su consulta y visualización en este u otros procesos de salud. 


\section{CONCLUSIONES}

Los registros de enfermería se consideran un instrumento efectivo para reflejar toda la información necesaria para poder coordinar y proporcionar unos cuidados de enfermería de máxima calidad al estar almacenados de forma integral.

El trabajo en equipo de Informáticos y Profesionales Sanitarios, optimiza el resultado final. $\mathrm{Se}$ consiguen unos registros de mayor calidad aunando pensamiento enfermero y tecnologías informáticas.

Se asegura la disponibilidad de toda la información de los procesos salud-enfermedad de un individuo, asegurando la confidencialidad (10).

El ciudadano y el profesional podrán tener acceso a los datos, en cualquier lugar del Departamento.

\section{BIBLIOGRAFÍA}

(1) Health Care Information and Management Systems Society (HIMSS) [Internet] The Healthcare Information and Management Systems Society (HIMSS) is the healthcare industry's membership organization exclusively focused on providing leadership for the optimal use of healthcare information technology (IT) and management systems for the betterment of healthcare. Annual Conferences Exhibition. March 1-4, 2010. Atlanta. Disponible en: http://www.himss.org

(2) David M. Stage 6 Hospitals: The Jouney and de Accomplishements. [acceso 20 mayo 2009]. Disponible en http://www.himssanalitics.org/docs/stageguithepaper.pdf

(3) Gordon M, Diagnóstico enfermero. Proceso y Aplicación. 3a ed. Barcelona: Doyma; 1996.

(4) Diagnósticos Enfermeros: Definición y Clasificación 2007-2008. NANDA Internacional. Madrid: Elsevier España, 2008.

(5) McCloskey Dochterman JC, Bulechek GM. Clasificación de Intervenciones de Enfermería (NIC). $4^{\circ}$ ed. Madrid: Elsevier España, 2004.

(6) Jonson M, Maas M, Morread S, Anderson M, Aquilino M, Belliner S. Clasificación de Resultados de Enfermería (NOC). 3a ed. Madrid: Elsevier España, 2004.

(7) Dowton J H. Escalas de riesgos de caídas, 1993.

(8) Carpenito LJ. Planes de cuidados y documentación clínica en enfermeria. Madrid: McGraw-Hill Interamericana, 2005

(9) Tucker SM y Cols. Normas para el cuidado de los pacientes. Procesos de Enfermería: Diagnósticos y resultados. Barcelona: Doyma, 1997.

(10) Ley Orgánica 15/1999, de 13 diciembre, de Protección de Datos. Boletín Oficial del Estado, 298, 11-12-1999) 


\section{ISSN 1695-6141}

Q COPYRIGHT Servicio de Publicaciones - Universidad de Murcia 